Maciej Ostrowski ${ }^{1}$

Uniwersytet Papieski Jana Pawła II w Krakowie

\title{
Theologie der heiligen Pilgerstätten - biblische und pastorale Aspekte
}

Am Ziel jeder Wallfahrt steht das Erreichen der heiligen Stätte. Dort erwartet der Pilger, Gott oder seinen Heiligen näher zu kommen. Er will Gott verehren und zugleich rechnet er damit, die gewünschte Gnade zu erhalten. Die heilige Stätte besteht im Christentum meistens aus einem sakralen Gebäude - einer Kirche oder Kapelle - manchmal auch aus einem größeren Raum, der gewöhnlich dieses Gebäude umgibt. Die heilige Stätte wird vom Pilger als Eigentum Gottes oder das Eigentum seiner Heiligen betrachtet, die in ihrem irdischen Leben seine Heiligkeit widerspiegelten. Der Pilger ist auch davon überzeugt, dass sich gerade dort, intensiver als an jedem anderen Ort, z.B. in der Pfarrkirche, die Wirkung der Gnade Gottes offenbart. Diese Überzeugung motiviert ihn, die Heimatstadt zu verlassen und sich auf den Pilgerweg zu begeben. Heilige Stätten existierten seit den Anfängen in fast jeder Religion. In diesem Beitrag soll die die christliche Theologie dieser Stätten in Anknüpfung an biblische Texte des Alten und Neuen Testaments charakterisiert

Maciej Ostrowski, Professor an der Päpstlichen Universität Johannes Paul II in Krakau, Polen, Leiter des Lehrstuhls für Pastoraltheologie in der Theologischen Fakultät; spezialisiert sich in Wallfahrts-, Tourismus- und Freizeitseelsorge, der Sekretär in dem Rat des Episkopats für Migranten, Touristen und Pilgern Seelsorge. 
werden. Anlässlich der Feierlichkeiten des Großen Jubiläums des Jahres 2000 veröffentlichte der Heilige Stuhl ein besonderes Dokument, welches sehr umfangreich die Theologie der heiligen christlichen Stätten entfaltete und zugleich Hinweise zur Seelsorge an diesen Stätten erteilte. ${ }^{2}$ Dieses Dokument wird uns als eine der Hauptquellen dienen. Aufgrund der enormen Spannweite des Themas, kann hier nur ein kurzer Abriss präsentiert werden.

\section{Die Entstehung heiliger Stätten dank der Initiative Gottes}

Wie anfangs gesagt, begibt sich der Pilger auf den Weg, um die heilige Stätte zu erreichen und dort Gott näher zu kommen. Über Jahrhunderte hinweg wurden an vielen geographischen Punkten der Welt zahlreiche Heiligtümer (Sanktuarien) erbaut. Sie entstanden dort, wo der Mensch besonders stark Gottes Anwesenheit empfand. Nach der Überzeugung der Menschen sollten sie günstige Bedingungen schaffen, um in eine geistige Gemeinschaft mit Gott einzutreten. Wie das oben zitierte Dokument des Heiligen Stuhles betont, war es nicht der Mensch, der den ersten Anstoß für Wallfahrtsorte gab. Gott selbst wollte dem Menschen nahe kommen, um ihm den Weg zu sich selbst zu eröffnen. So schreibt der hl. Johannes in seinem Brief: „Wir wollen lieben, weil er uns zuerst geliebt hat" $(1$ Joh 4,19) und „seinen einzigen Sohn in die Welt gesandt hat” (1 Joh 4, 9). Die Pilger entdecken im Heiligtum (Sanktuarium) „das Zeichen des unter uns lebenden Christus [...] eine Initiative der Liebe des lebendigen Gottes für Menschen unternommen."3

Gott offenbarte sich Moses im brennenden Dornbusch, um seine Pläne gegenüber dem Auserwählten Volk zu verkünden. In seiner Sorge um die von der Sklaverei betrübten Israeliten berief er Moses zum Füh-

2 Päpstlicher Rat der Seelsorge für die Migranten und Menschen unterwegs, Heilige Stätten - Erinnerung, Gegenwart und Prophezeiung des lebendigen Gottes, herausgegeben am 8. Mai 1999, http://www.vatican.va/roman_curia/pontifical_councils/migrants/documents/rc_pc_migrants_doc_19990525_shrine_it.html (30.12.2013). sa 29,7$)$.

Ebd, Nr. 5. die Urkunde beruft sich auf Theodoret aus Kyrrhos (Historia Religio- 
rer, der dank der Kraft aus der Höhe das Volk aus Ägypten herausführen sollte. Gerade diese Stätte wurde durch die Anwesenheit Gottes zum „Gelobten Land“.

Deshalb verlangte Gott von Moses, seine Schuhe abzulegen, da der Boden, wo er stehe, heiliger Boden sei, der Boden heiliger Anwesenheit (vgl. Ex 3, 1-10). Diese Anwesenheit Gottes und ihre Wirkung heiligt jedes Heiligtum (Sanktuarium) und lässt jeden Pilger sich mit Hochachtung vor dem Heiligen verbeugen.

Andererseits sucht der Mensch selbst nach Gott. Dies wird überzeugend durch die Worte des Psalmisten ausgedrückt: „Gott du mein Gott, dich suche ich [...]. Darum halte ich Ausschau nach dir im Heiligtum, um deine Macht und Herrlichkeit zu sehen" (Ps 63, 2-3).

Der Mensch sucht auf der Erde nach einem Platz, der im die Begegnung mit Gott und seiner Erkenntnis erleichtert. Die göttlichen Wege „aus der Höhe“ treffen sich mit menschlichen „irdischen“ Wegen, die Absichten Gottes mit den menschlichen Sehnsüchten.

\section{Die Heilige Stätte als Eingang in die göttliche Ruhe}

Die Bibel schildert oft Wanderungen zu verschiedenen Zielen. Gott selbst bestimmt am häufigsten das Ziel für den Menschen. Das Erreichen dieses Ziels bedeutet die Erfüllung der göttlichen Absichten. Das Erreichen jedes irdischen Ziels ist mit Erholung, Zufriedenheit und Freude verbunden. Im biblischen Kontext ist das Erreichen des von Gott bestimmten Ziels mit einer tiefen geistlichen Erfahrung verbunden: das Empfinden der Gottes Nähe, die Empfindung der Fülle des Lebens, das erneute Entdecken des Lebenssinnes.

Betrachten wir an dieser Stelle den Exodus - die Geschichte von Israel. Das Ziel der Wanderung des auserwählten Volkes aus Ägypten war das Erreichen des von Gott versprochenen Gelobten Landes. Aus menschlicher Perspektive sollte dieses Land zum Land der Ruhe nach den langen Wegstrapazen durch die Wüste werden. Aber es wurde in erster Linie gemäß dem Psalmwort zum Land der Ruhe Gottes als dessen Eigentum (vgl. Ps 95, 11). Gott wollte die Ruhe mit seinem Volk teilen. Das Wesen 
dieser Ruhe lag nicht nur in der Erholung von den Strapazen der vierzigjährigen Wanderung durch die Wüste, sondern vor allem in der Erfahrung der Freude über das Erreichen des von Gott bestimmten Ziels, im Genießen des Friedens und der endgültigen Besiegelung des göttlichen Versprechens. Das Auserwählte Volk konnte sich schlussendlich überzeugen, dass Gott es nicht verlassen hatte, und sein Schutz über das Volk weiter bestand. Die durch die Wüste Wanderndernden haben diese Ruhe als ein Eingehen in eine tiefere und neue Gemeinschaft mit ihrem Gott Jahwe verstanden. Die Ruhe im Land Kanaan wurde für die Israeliten zur Belohnung für ihre Bundestreue.

Urbild aller gegenwärtigen heiligen Stätten wurde die heilige Stadt Jerusalem ${ }^{4}$ und dort besonders der Tempel. Der Psalmist sagt ausdrücklich über den Tempel in Jerusalem: „Erheb dich Herr, komm an den Ort deiner Ruhe, du und deine machtvolle Lade! [...] Das ist für immer der Ort meiner Ruhe, hier will ich wohnen" (Ps 132, 8. 14). Der Tempel und ganzJerusalem waren die durch das Recht gebotenen Wallfahrtsziele für die Israeliten (vgl. Dtn 15, 16-17; Ex 34, 24). Diese Stätten wurden für sie gewissermaßen zum neuen Gelobten Land, wo Gott seine Ruhe mit den Pilgern teilte. Hier standen die Pilger in Gottes Anwesenheit und empfanden darüber eine tiefe Freude. Hierzu schreibt der Prophet Jesaja: „Die vom Herrn Befreiten kehren zurück und kommen voll Jubel nach Zion. Ewige Freude ruht auf ihren Häuptern. Wonne und Freude stellen sich ein, Kummer und Seufzen entfliehen " (Jes 35, 10). Die heilige Stadt und ihr Tempel waren für die Israeliten sowohl ein Zeugnis der unaufhörlicher Liebe und des Gottes Schutzes, als auch das Zeugnis der Beständigkeit des mit ihm geschlossenen Bundes.

Zugleich waren sie auch Stätten der ständigen Erneuerung und Aktualisierung dieses Bundes.

Hier verpflichtete sich das Volk immer wieder dazu, in Treue gegenüber Gott und sein Recht zu leben. Der Raum der Stadt und des Tempels trug ein sakrales Brandmal und war voll geheiligter Macht. Jerusalem wurde zum Zentrum der Heiligung des Menschen. Der Pilger kehrte nach Hause geistlich erneuert und geheiligt zurück.

${ }_{4}$ Vgl. Sir 36, 12. 
Die Pilger, die sich heute zu heiligen Stätten begeben, erwarten von ihnen, dort eine besonders intensive Nähe Gottes zu erleben. Von der „Kondensation“ der Anwesenheit Gottes im heiligen Raum zeugen die von den Pilgern erfahrenen Gnaden. Die meisten der Gaben Gottes sind nur den Empfängern bekannt, aber es gibt auch manche, wennglich wenige, die offiziell als Heilungen, Wunder und Offenbarungen anerkannt wurden. Wenn die Pilger nach den Strapazen des Weges das Ziel erreichen, sehnen sie sich nach Erholung. Die heilige Stätte wird für sie mit Sicherheit zur Oase der Erholung der physischen Kräfte. Viel wichtiger ist jedoch die geistige Erholung - der Aufenthalt in Gottes Anwesenheit. Gott selbst wird zum Ort der Ruhe für die an ihn Glaubenden. Hier ist Zeit für ein Abstandnehmen von Alltagsproblemen, die den Menschen belasten, Zeit für eine Vertiefung im Gebet und für das Nachdenken über den Sinn des Lebens. In der heutigen Welt, in der belastende Eindrücke und Hektik mehr als in anderen Epochen überhandnehmen, erscheint die Zeit der Ruhe und geistiger Erholung als besonders wertvoll. ${ }^{5}$

\section{Die Ankündung der eschatologischen Glückseligkeit}

An dieser Stelle sei nochmals auf die Schilderung Jerusalems und seines Tempels zurückgekommen. Der Berg, auf dem der Tempel des Herrn stand, wurde für das auserwählte Volk zum Symbol der Erlösung. Psalm 15 stellt die Frage: „Herr, wer darf Gast sein in deinem Zelt, wer darf weilen auf deinem heiligen Berg?" (Ps 15, 1), worauf gleich die Antwort folgt: „der makellos lebt und das Rechte tut“(Ps 15, 2). Der Psalmist zeigt demzufolge, dass nur derjenige, der Gottes Recht einhält, die Erlösung erlangt. Die Pilger, die den Berg des Tempels in Jerusalem betraten, konnten den Vorgeschmack der zukünftigen Glückseligkeit mit Gott erleben, indem sie im Tempel feierten. Der 84. Psalm spiegelt die Glückseligkeit der im Tempel verweilender Menschen wider. Dort lesen wir: „Wohl denen, die wohnen in deinem Haus, die dich allezeit

1996, S. 45.

Vgl. M. Ostrowski, Duszpasterstwo wobec problemu wolnego czasu człowieka, Kraków 
loben“ (Ps 84,5). Die Tore des Tempels symbolisierten den Übergang zu einer anderen, überirdischen Glückseligkeit. Sie wurden gewissermaßen zu Toren des Himmels selbst. Auf der einen Seite blieb das, was wir mit dem Begriff „Profanum“ bezeichnen. Auf der anderen Seite befand sich das "Sacrum“. Hier ließe sich noch eine andere biblische Szene anführen, nämlich der prophetische Traum Jakobs. Der Prophet erblickte im Traum eine Leiter, die die Erde mit dem Himmel verband. An diesem Ort stellte er ein Steinmal auf, Vorausbild des zukünftigen Tempels. Er sagte über diesen Ort: „Wie Ehrfurcht gebietend ist doch dieser Ort! Hier ist nichts anderes als das Haus Gottes und das Tor des Himmels" (Gen 28, 17). Das Gelobte Land Jerusalem und sein Tempel wurden zu eschatologischen Symbol der Freude über den ewigen Aufenthalt in der Gottes Nähe.

Es lohnt sich hier, andere biblische Aussagen aus dem Buch Jesaja anzuführen: „Am Ende der Tage wird es geschehen! Der Berg mit dem Haus des Herrn steht fest gegründet als höchster der Berge, [...] zu ihm strömen alle Völker. [...] Sie sagen: Kommt, wir ziehen hinauf zum Berg des Herrn und zum Haus des Gottes Jakobs “ (Jes 2, 2-3) Der Prophet verweist hier auf die eschatologische Zeit, wenn sich nicht nur Israel, sondern auch die Erlösten aus allen Völkern im Reich Gottes versammeln werden. Der Tempelberg und der Tempel selbst - Ziel der Wallfahrten - stellt das Ziel der endgültigen Wallfahrt der ganzen Menschheit dar. An einer anderen Stelle sagt der Verfasser des Briefes an die Hebräer: „, Denn wir haben hier keine Stadt, die bestehen bleibt, sondern wir suchen die künftige" (Hebr 13, 14). Das Ziel der Pilgerfahrt symbolisiert damit das ewig bestehende Haus im Himmel, wo sich Gott nach seinem ewigen Ratschluss wünscht, mit allen Menschen in ewiger Gemeinschaft verneigt zu sein. ${ }^{6}$

Jede heilige Stätte wird auf diese Weise zum Symbol des „,neuen Gelobten Landes“ - „eines neuen Himmels und einer neuen Erde“ (Offb 21, 1).

Vgl. Discorso di Giovanni Paolo II ai participanti al I Congresso Mondiale della Pastorale dei Santuari e dei Pellegrinaggi, 28 febbraio 1992, http://www.vatican.va/holy_father/ john_paul_ii/speeches/1992/february/documents/hf_jp-ii_spe_19920228_pastorale-santuari_it.html (25.03.2014). 
Für die Gläubigen wird im Heiligtum das ewige Verweilen mit Gott im Himmel, die ewige Ruhe in seinem Reich in gewisser Weise vorweggenommen. In dem schon zitierten Dokument des Heiligen Stuhles können wir lesen: „Im Sanktuarium bestätigt sich die eschatologische Dimension des christlichen Glaubens, das heißt sein Streben nach der Vollkommenheit des Reiches"; deswegen erfüllt es auch eine prophetische Funktion, als ein Zeichen der Hoffnung ${ }^{7}$, die den Menschen zum Erreichen des definitiven und endgültigen Zieles auffordert: der „endgültigen und definitiven Heimat", „des himmlischen Jerusalems“, welches die ewig bestehende Gemeinschaft mit Gott ist. Es hilft uns zu „erkennen, wohin wir gehen, an welches Ziel sich unsere Pilgerfahrt im Leben und in der Geschichte wendet." ${ }^{8}$

Zum besonderen Moment der Antizipation der eschatologischen Wirklichkeiten wird die Liturgie, die an heiligen Stätten gehalten wird. Hierzu sagt das Zweite Vatikanische Konzil: „Die irdische Liturgie verleiht uns den Vorgeschmack der Teilnahme an der himmlischen Liturgie, die in der heiligen Stadt Jerusalem gehalten wird, wo Christus zu rechten Gottes sitzt als Diener des Gottes Hauses und des wahren Tempels (vgl. Offb 21, 2; Kol 3, 1; Hebr 8, 2).“9

\section{Die Identität und Würde}

Erneut soll noch einmal an die biblischen Bilder angeknüpft werden, die im vorangehenden Abschnitt beschrieben wurden. Nach der langen Zeit der Sklaverei, der Ausbeutung durch Fremde, des Heimatentzugs und später der Unsicherheit verbunden mit dem Umherirren in der Wüste erreichten die Israeliten das Gelobte Land. Als ein Volk genossen sie hier Freiheit und Souveränität. Sie befanden sich im eigenen Land, das ihnen vom Herrn geschenkt worden war. Sie selbst wur-

Heilige Stätten..., zitiertes Dokument, Nr. 16.

Ebd., Nr. 13.

Sacrosanctum concilium, Nr. 8. 
den zu Herren dieses Landes und konnten dadurch wieder neu ihre Würde und Identität empfinden. ${ }^{10}$

Zum Zentrum des Gelobten Landes wurden die heilige Stadt Jerusalem und der Tempel des Herrn. Dort neigte sich Gott seinem Volk zu, um sich mit ihm niederzulassen. Vielmehr lud Gott selbst sein Volk ein, sich mit ihm niederzulassen ${ }^{11}$. Diese Räume waren für das auserwählte Volk eine Garantie seines Bestehens und seiner Identität. Es ging hier nicht nur um ein bestimmtes sichtbares Gebiet - eine Stadt mit ihrer Bebauung, einen Tempel mit seinen Mauern - sondern vor allem um Gott selbst, dessen Anwesenheit im Tempel und in der Stadt Raum einnahm. Gottes Anwesenheit am heiligen Ort selbst war es, die das in viele Richtungen zerstreute Volk vereinigte und ihm Schutz bot. Der Psalmist vergleicht die Anwesenheit von Jahwe mit einem Felsen, an dem das Haus des Herren befestigt ist. Auf diesem Felsen sind die Geschlechter Israels gebaut (vgl. Ps $46,1-8)^{12}$.

Ezechiel sagt, der Herr habe die Zerstreuung seines Volkes zugelassen, aber jetzt versammle er das Volk und er gebe ihm das Land zum Besitz; Gott selbst werde unter ihm zum Tempel und so werden die Israeliten „mein Volk sein und ich werde ihr Gott sein“" (Ez 11, 14-20).

Ähnlich wie die Israeliten im Gelobten Land und in der heiligen Stadt ihre nationale Einheit (Identifikation) und Würde erfahren haben, so öffnet sich auch vor den Pilgern, die zu den heiligen Stätten wallfahrten, eine Chance, ihre christliche Identität neu zu entdecken.

Dort nähern sie sich Gott im Gebet und Sakramenten, hören auf intensivere Weise das Wort Gottes, werden in Christus ,auferbaut " - und intensivieren die Bindung an ihn als lebendige Steine, als lebendiger Tempel (vgl. 1 Petr 2, 5. 9). Pilgern wird oft als Exerzitien unterwegs bezeichnet, und die Seelsorge im Heiligtum fördert die Glaubensvertiefung und die Rückkehr zu den Wurzeln. ${ }^{13}$ Auf diesem Weg wird die christliche Identität gestärkt. Andererseits fördern die gleichen Erlebnisse eine tiefe Bekeh-

\footnotetext{
Vgl. Jos 24, 1-28.

Vgl. Offb 21, 3.

Dtn 32, 18; Ps 18, 3.

Heilige Stätten..., zitiertes Dokument, Nr. 10.
} 
rung, eine neue Erfahrung des Lebens als Kind Gottes. Auf diese Weise wird während der Pilgerfahrt die Überzeugung der christlichen Würde belebt. Die heilige Stätte symbolisiert für die heutigen Pilger - ähnlich wie bei den Israeliten Jerusalem und sein Tempel - die ewigen, unvergänglichen, evangelischen Werte, die den dauerhaften Bezugspunkt unseres Glaubens und irdischer Bestrebungen bilden.

\section{Ort des Aufbauens der ekklesialen Gemeinschaft}

Kommen wir nochmals auf sie oben zitierten Worte des Propheten Jesaja zurück: „Am Ende der Tage wird es geschehen: der Berg mit dem Haus des Herrn steht fest gegründet als höchster der Berge [...]. Zu ihm strömen alle Völker" (Jes 2, 2-3). Wie bereits betont war der jüdische Tempel ein Symbol der Einheit des auserwählten Volkes, Symbol des einen Kultes, der auf den einzigen Gott Jahwe orientiert war. Die Einheit des auserwählten Volkes wurde durch das Pilgern zu einem Tempel gebaut. Der Text des Propheten knüpft an diese Tradition des Pilgerns an, die vom Recht geboten wurde. Gleichzeitig verweist sie auf die künftige messianische Zeit. Der Berg Zion und Jerusalem symbolisieren das religiöse Zentrum aller Völker. Zu diesem Zentrum wird später die Kirche Christi. An Pfingsten versammelten sich auf der Jerusalem-Pilgerfahrt ,Juden, fromme Männer aus allen Völkern unter dem Himmel“ (Apg 2, 5) und sie alle wurden auf wundervolle Weise durch die Kraft des Heiligen Geistes vereinigt. In diesem Moment bekam die alttestamentliche Pilgerfahrt eine neue Qualität. Seit Pfingsten pilgern die Gläubigen aus allen Völkern der Welt, um sich in einer Kirche, um sich in dem neuen Tempel - in Christus selbst - zu versammeln. ${ }^{14}$

Jede heilige Stätte der Christen steht offen für alle neu angekommene Pilger aus allen Völkern. Sie wird nicht nur zu einem Raum der menschlichen Versammlung, sondern auch der geistigen Vereinigung, besonders wenn dort die Eucharistie gefeiert wird. Die Heiligtümer werden zu Zentren, in die Pilger aus verschiedenen Weltrichtungen kommen, die

14 Vgl. Joh 2, 19-21, Apg 21, 22. 
sich voneinander durch die Sprache, Kultur, Alter und Familienstand unterscheiden. In einer geschichtlichen Epoche, in der durch die Verkehrserleichterungen neue Möglichkeiten des schnellen Ankommens in viele Gegenden der Welt eröffnet werden, gewinnen die heiligen Stätten einen internationalen und überregionalen Charakter. Trotz vieler Unterschiede zwischen den Pilgern, verbindet sie der ähnliche Wunsch nach einer intensiveren Begegnung mit Gott und dem Erhalt seiner Gnaden. Sie beten als eine Kirche Christi vor demselben Gott. Sie bauen eine Gemeinschaft um Christus, der das Haupt ist. Sie nehmen an denselben Sakramenten, besonders an der Eucharistie, teil. Im gegenseitigen Kontakt folgt der Austausch verschiedener Traditionen, z.B. des Gebetsstils, der Liturgie oder unterschiedlicher religiöser Bräuche. Die Pilger haben am heiligen Ort gewissermaßen die Gelegenheit einer greifbaren Erfahrung der Katholizität und Universalität der Kirche. Diese Kirche ist, obwohl sie aus vielfältigen, sich voneinander unterscheidenden Gliedern besteht, die gleiche, einzige Kirche, zu der jeder von ihnen individuell gehört. Sie ist eine Gemeinschaft, in der wir alle zum Herrn pilgern. Derartige Erlebnisse haben einen positiven Einfluss auf die Identifizierung mit der Kirche. Wir wiederholen also, das was schon betont wurde, dass am heiligen Ort die Erfahrung der christlichen Identität auf gemeinschaftlicher Ebene geschieht.

Wie das zitierte Dokument des Päpstlichen Rates betont, ist das Sanktuarium der Ort, wo die ekklesiale Gemeinschaft auferbaut wird. Die Pilger „werden zum Heiligtum aus lebendigen Steinen, und dadurch sind sie in der Lage, die erneute Erfahrung der Gemeinschaft des Glaubens und der Heiligkeit, welche die Kirche ist, zu erleben“. Weiter besagt das Dokument, dass dank der Wirkung des Heiligen Geistes die Pilger den Reichtum und die Vielfältigkeit der Kirche erkennen können, und dadurch sich auch auf andere Personen zu öffnen vermögen, für sie Verantwortung übernehmen, und für andere den Dienst übernehmen, dank der Gabe, die jeder von ihnen besitzt. ${ }^{15}$ Jede heilige Stätte trägt also in sich die Potentialität einer tieferen Gestaltung der kirchlichen Gemeinschaft und der Verantwortung der Gläubigen für die Kirche.

\footnotetext{
15 Heilige Stätten..., zitiertes Dokument, Nr. 12.
} 


\section{Ort des Gebets, Sühne und Erneuerung}

Man sollte schließlich auch einen der häufigsten Gründe des Pilgern zu heiligen Stätten erwähnen - der Mensch pilgert nämlich, um sich mit Gott im Gebet zu vereinigen, ihn zu loben und Opfer darzubringen. Die Jerusalempilger sangen beim Betreten der Stufen des Tempels freudige Lieder. In einem der Gradualpsalmen ${ }^{16}$ heißt es: „Dorthin ziehen die Stämme hinauf, die Stämme des Herrn, wie es Israel geboten ist, den Namen des Herrn zu preisen“ (Ps 122, 4). In einem anderen Psalm lesen wir: „Ich will Opfer darbringen in seinem Zelt, Opfer mit Jubel; dem Herrn will ich singen und spielen" (Ps 27, 6). Die Zeit des Aufenthalts im Tempel war die Zeit der Dankbarkeit für die von Gott erfahrenen Gaben und die Zeit der Darbringung der Opfer. Dieser Ort wurde zum Zentrum des Kultes für das ganze Volk und zum Mittelpunkt der Verehrung Gottes, sozusagen zum Herzen der Vereinigung mit ihm.

Das Dokument des Päpstlichen Rates über das Pilgern bezeichnet die heiligen Stätten als Zeichen des Gebets, Sühne und Erneuerung. ${ }^{17}$ In besonderer Weise wird dort das religiöse Leben der Kirche intensiviert. Hier kann sich ihre Kultus- und Gebetsströmung entwickeln. Wie das Auserwählte Volk im Gelobten Land seine Freiheit erneut erfahren hatte, so haben auch die im Sanktuarium verweilenden Pilger die Chance, die geistige Freiheit zu genießen - zur Sühne und Erneuerung des Lebens zu finden und eine neue Erfahrung des Lebens als Kind Gottes zu machen. Durch das Hören auf Gottes Wort und eine intensive Teilnahme an den Sakramenten werden sie zu Erneuerung des Lebens gerufen - besonders im Sakrament der Beichte und der Eucharistie - sie bekommen dafür auch die entsprechende Hilfe angeboten. Der Aufenthalt der Pilger im Sanktuarium wird somit zu einer Verpflichtung für die Zukunft - zur Verpflichtung eines Lebens in Gottesfurcht. ${ }^{18}$

16 Die Lieder der Stufen wurden bei dem Betreten des Tempels in Jerusalem gesungen.

17 Päpstliche Rat der Seelsorge für die Migranten und Menschen Unterwegs, Die Pilgerfahrt zum großen Jubiläum 2000, 25 April 1998, N. 7, http://www.vatican.va/roman_curia/ pontifical_councils/migrants/documents/rc_pc_migrants_doc_19980425_pilgrimage_ge.htm (30.12.2013).

18 Ebd. 7. 
Gleichzeitig opfern die Pilger im Heiligtum die Mühsale und Unbequemlichkeiten des Weges auf. Ein Theologe hat es einmal so bezeichnet: „sie beten mit den Füssen. "19 Ihre körperlichen Anstrengungen, besonders die während einer Fußwallfahrt, vertiefen und verstärken gewissermaßen die Gebete der Pilger: ihre Danksagungen, Bitten und ihre Sühne.

\section{Freude der Pilger}

Wie wir schon bemerkten, stellt das Erreichen des Zieles der Pilgerfahrt für die Pilger eine Quelle der Freude ${ }^{20}$ dar. Je größer die Strapazen und Erfahrungen des Weges waren, desto stärker wird das Erleben der Freude an der heiligen Stätte sei. Am Ziel jeder Pilgerfahrt werden alle Strapazen vergessen. Zurück bleibt nur das glückliche Bewusstsein, dass alle Sehnsüchte in Erfüllung gegangen sind. Die Freude des Pilgers ergibt sich aus der Möglichkeit des Verweilens in der Nähe des Herrn, aber auch aufgrund der Gemeinschaft mit den Glaubensbrüdern. Die Gradualpsalmen sind von der Freude erfüllt, die vom Betreten des Tempels herrührt, in dem Gott anwesend ist. ${ }^{21}$ In einem von ihnen steht: „Ich freute mich, als man mir sagte: Zum Haus des Herrn wollen wir pilgern“ (Ps 122, 1). Ein anderer lobt die Gemeinschaft der Brüder; „Seht doch, wie gut und schön ist es, wenn Brüder miteinander in Eintracht wohnen“ (Ps 133, 1). Darin liegt auch die Freude von der im Sanktuarium erhaltener Gabe der Vergebung und Versöhnung mit Gott. Sie entsteht im Herzen, das von Sünde befreit und neu von Gnade erfüllt wird (vgl. Ps 130). Derartige Freude ist gemäß dem Worte Christi dauerhaft, und keiner kann sie uns wegnehmen (vgl. Joh 15, 11;16, 23). Das mehrmals angeführte Dokument des Päpstlichen Rates sagt hierzu: „Im Sanktuarium lernt das Volk Gottes, eine «Kirche der Freude» zu sein“. Das Heiligtum ist, wie schon erwähnt wurde,

19 E. Mielenbrink, Beten mit den Füßen. Über Geschichte, Frömmigkeit und Praxis von Wallfahrten, Düsseldorf 1993.

20 Vgl. ebd, S. 55.

21 Die Psalmen 120-134. 
eine Erinnerung an Gottes Nähe und seine Wirkung unter den Menschen. Diese Anwesenheit gibt uns die Sicherheit, dass wir trotz der Strapazen, die mit dem irdischen Leben verbunden sind und trotz der Leiden und Erfahrungen, die die Pilgerfahrt in der Welt mit sich bringt, „die Morgenröte der Zukunft [sehen], dass das Königreich schon da ist und deswegen auch unsere Herzen von Freude, Vertrauen und Hoffnung erfüllt sein [können].“22 Die Pilger können auch heute durch das von Freude erfüllte Gebet die alttestamentlichen Graudualpsalme singen.

Unser Beitrag berücksichtigte mit Sicherheit nicht vollständig den umfangreichen Themenbereich der Theologie der heiligen Stätten. Er bemühte sich nur, bescheidene pastorale Schlussfolgerungen zu formulieren. Wir können in diesem Zusammenhang eine wichtige Folgerung aufstellen: In den zunehmend entchristlichten Ländern der heutigen Welt, besonders Europas, in denen der Zerfall der Kirchenstruktur fortschreitet, haben die heiligen Stätten die Möglichkeit, nicht nur Zentren der Erneuerung des Glaubens, sondern auch der Festigung und sogar direkt seines Wiederaufbaus zu werden. Als Zeichen der neuen Welt und des neuen Himmels geben sie uns Hoffnung auf die Neubelebung des christlichen Lebens.

22 Heilige Stätten..., zitiertes Dokument, Nr. 15. 


\section{Summary}

\section{Theology Of Holy Places - Biblical and Pastoral Aspects}

The article focuses on aspects of theology of shrines, which are destinations of Christian pilgrimages. The author bases on biblical texts, particularly on these concerning the Promised Land and the Holy City of Jerusalem with its temple, further on documents of the Pontifical Council for the Pastoral Care of Migrants and Itinerants. Each earthly shrine is an expression of God's love for a human. On the one hand, God's initiative is to create a convenient place for His encounter with a man, on the other hand, a human himself searches for such a place. A shrine is a venue, where a man "has rest in God", enters into a community with Him. Simultaneously, it anticipates future communion with God in the eternity - "eternal rest in God" after efforts of the earthly pilgrimage. It is there, where a human being will achieve complete happiness and joy. Like in the past, the Promised Land and the temple in Jerusalem were places for creating unity and identity for Israelites, nowadays shrines are means of creating and enhancing Church community, which embraces comers from all over the world. The article emphasis the fact that present shrines are not only places of intensive prayers, great liturgical celebrations, but also centres where people offer their spiritual sacrifices, do penance, further where sinners convert to God and each pilgrim renews spiritually.

Keywords: shrine, pilgrimage, theology, Bible, pilgrimage ministry

\section{Theologie der heiligen Pilgerstätten - biblische und pastorale Aspekte}

Der Artikel konzentriert sich auf ausgewählte Aspekte der Theologie der Sanktuarien, der Orte, die Ziel christlicher Pilgerfahrten sind. Der Autor stützt sich auf biblische Texte, besonders betreffend das Gelobte Land und die Heilige Stadt Jerusalem mit seinem Tempel, sowie Dokumente des Päpstlichen Rates für die Seelsorge der Migranten und Menschen unterwegs. Jedes irdische Sanktuarium drückt an erster Stelle die Liebe Gottes an den Menschen aus. Gott schafft mit seiner Initiative einen günstigen Platz für ein näheres Treffen mit dem Menschen. Auf der anderen Seite sucht der Mensch selbst einen solchen Platz. Das Sanktuarium ist ein Ort, in dem der Mensch „in Gott ruht” - kommt mit ihm in eine nahe Gemeinschaft. Gleichzeitig kündigt es die künftige Vereinigung mit Gott in Ewigkeit an - „ewige Ruhe in Gott " nach den Strapazen der irdischen Pilgerfahrt. Dort wird der Mensch die Fülle der Freude und das Glück erreichen. So wie das Gelobte Land und der Tempel von Jerusalem für die Israeliten das Zentrum der Schaffung der Einheit und der Identität des auserwählten Volkes waren, so sind heute Sanktuarien das Werkzeug der Erschaffung und Befestigung der Gemeinschaft der Kirche, an der sich Ankömmlinge aus allen Himmelsrichtungen 
drängen. Der Artikel unterstreicht, dass die heutigen Sanktuarien nicht nur Orte intensiven Gebets sind, der Blütezeit der Liturgie, sondern auch Zentren der Darbringung von geistigen Opfern, Buße und Bekehrung der Sünder, sowie geistiger Erneuerung eines jeden Pilgers.

Schlüsselbegriffe: Heiligtum, Wallfahrt, Theologie, Bibel, Wallfahrtseelsorge

\section{Bibliographie}

Dec I., Człowiek jako pielgrzym. O pielgrzymowaniu w aspekcie filozoficzno-teologicznym na tle pielgrzymowania do Sanktuarium Matki Bożej Pocieszenia w Leżajsku, „Peregrinus Cracoviensis“ Bd. 7 (1999), S. 61-72.

Majewski M., Mieszkanie Chwały: teologia sanktuarium Izraela na pustyni (Wj 25-31; 35-40), Kraków 2008.

Ostrowski M., Sanktuarium w teologiczno-duszpasterskiej perspektywie, [in:] Zawierzenie Bożemu Miłosierdziu w łagiewnickim sanktuarium. Materiały z sympozjum, Kraków 2003, S. 41-62.

Ostrowski M., Teologia pielgrzymowania, „Polonia Sacra” 12 (2003) 1, S. 277-294.

Pontifical Council for the Pastoral Care of Migrants and Itinerant People, The shrine a privileged place for a meeting between God and his people, a pilgrim in time. Precedings of the XIV Plenary Meeting 23-25 June 1999, Rome 1999.

Pontificio Consiglio della Pastorale per I Migranti e gli Itineranti, Santuario Nostra Signora di Montserrat, Il Santuario, spazio per un'accgilenza fraterna e universale, Città del Vaticano 2002.

Siudy T., Sanktuaria maryjne w postudze ewangelizacyjnej Kościoła, [in:] Geografia i sacrum, t. 2, pod red. B. Domańskiego, S. Skiby, Kraków 2005, s. 290-301.

Wolf N., Wohin pilgern wir? Alte Wege und neue Ziele, Reinbek bei Hamburg 2011.

Tłumaczenie na język niemiecki: Marta Krzanowska Korekta językowa niemiecka: Michaela Hastetter 